\title{
Treatment of heavy menstrual bleeding with a new combination of estradiol valerate and dienogest
}

This article was published in the following Dove Press journal:

Open Access Journal of Contraception

I November 2010

Number of times this article has been viewed

\author{
Luis Bahamondes \\ Ilza Monteiro \\ Arlete Fernandes \\ Human Reproduction Unit, \\ Department of Obstetrics and \\ Gynecology, School of Medical \\ Sciences and National Institute of \\ Hormones and Women's Health, \\ University of Campinas, Campinas, \\ Brazil
}

Correspondence: Luis Bahamondes Caixa Postal 6I8I, I3084-97I,

Campinas, SP, Brazil

Tel +55 I 932892856

Fax +55 I9 32892440

Email bahamond@caism.unicamp.br

\begin{abstract}
The first combined oral contraceptive (OC) was launched in the US 50 years ago and was followed by another formulation introduced in Germany one year later. The most common estrogen component in current formulations is ethinylestradiol; however, many concerns have been raised with respect to this estrogen. Although the natural estrogen produced by the ovary, 17-beta estradiol, is the most potent of the estrogens, it is poorly absorbed orally, and previous attempts to use it in combined OCs have been unsuccessful due to the occurrence of irregular bleeding. Recently, a new combined OC was developed containing a natural estrogen, estradiol valerate, and a new progestin, dienogest, in a dynamic 26-day, four-phasic (estrogen stepdown and progestin stepup) scheme of administration. In clinical trials, its contraceptive performance was excellent, with good cycle control and bleeding patterns compared with other combined OCs or with placebo. This review focuses predominantly on the use of an estradiol valeratedienogest combined OC for the treatment of heavy menstrual bleeding. The findings of two large, randomized, controlled trials have shown that this combined OC constitutes an effective treatment for women with heavy menstrual bleeding, representing a new therapeutic option to reduce menstrual blood loss. Further studies are necessary to confirm these data.
\end{abstract}

Keywords: dienogest, estradiol valerate, heavy menstrual bleeding, menorrhagia, contraception

\section{Introduction}

Since the first combined oral contraceptive (OC) was introduced 50 years ago, many different compounds and associations have been formulated, and the main estrogen component of almost all the combined OCs that have been developed has been ethinylestradiol. Nevertheless, several studies have found associations between the use of ethinylestradiol and venous thromboembolism. Therefore, the initial objectives of studies carried out to develop new combined OCs were to decrease both the amount of synthetic estrogen and the components responsible for provoking breakthrough bleeding. A reduction in the risk of venous thromboembolic events was achieved by the introduction of ethinylestradiol doses below $35 \mu \mathrm{g}$.

Because combined OCs with low and ultralow doses of ethinylestradiol $(20,15$, or $10 \mu \mathrm{g})$ are also associated with high discontinuation rates due to breakthrough bleeding or other menstrual disturbances, studies have been conducted with natural estrogens, specifically estradiol, in an attempt to reduce these risks. ${ }^{1-4}$ This strategy was followed by the addition of new progestogens with more favorable clinical profiles in the form of combined oral C-21 derivatives (cyproterone acetate) or C-19-nortestosterone derivatives (desogestrel and gestodene), followed later by a derivative of spironolactone (drospirenone). Progestogen components with antiestrogenic and androgenic activity 
have also been shown to contribute towards increased risk of stroke and ischemic heart disease..$^{5-7}$

Recent pharmacologic studies have focused on the replacement of the synthetic estrogen, ethinylestradiol, by estradiol, including 17-beta estradiol $\left(17 \beta-\mathrm{E}_{2}\right)$, or by estradiol valerate. In clinical studies, compounds containing combined oral $17 \beta-\mathrm{E}_{2}$ in monophasic and biphasic combined OCs have been found to result in poor cycle control, prolonged bleeding, and increased days of bleeding and spotting, with high discontinuation rates. ${ }^{8-12}$ In addition to the introduction of $17 \beta-\mathrm{E}_{2}$, other initiatives have included the use of estradiol valerate, which is identical to endogenously produced $17 \beta-\mathrm{E}_{2}$ as the estrogenic component of combined OCs. In fact, $1 \mathrm{mg}$ of estradiol valerate is equivalent to $0.76 \mathrm{mg}$ of estradiol based on molecular weight. Possible causes for the poor cycle control found with $17 \beta-\mathrm{E}_{2}$ may be an inappropriate estradiol/progesterone balance and/or an insufficient dose of estradiol. These issues have been addressed in several studies using different doses and regimens to determine the best system for steroid use. It has been speculated that in monophasic estradiol-progestin combined OC preparations, the progestin used in the formulations under investigation (eg, norethisterone acetate, desogestrel, or cyproterone acetate) and estradiol are metabolized slowly because the $17 \alpha$-ethinyl group prevents fast absorption and this may affect the overall bleeding profile. , $^{8,9,13}$

Early attempts to use estradiol in combined OCs were unsatisfactory in terms of bleeding control. The estradiol molecule is as effective as ethinylestradiol in the endometrium, but oral bioavailability is higher with ethinylestradiol (mean approximately $45 \%$, interindividual variation $20 \%-65 \%)^{14}$ than with estradiol. Furthermore, 17 $\beta$-hydroxysteroid dehydrogenase metabolizes estradiol into its less active metabolites. Thus, estradiol is metabolized extensively in the bowel wall and liver, and enters the systemic circulation mostly as estrone, estrone sulfate, and estrone glucuronide. ${ }^{15}$ For this reason, the bioavailability of estradiol is less than $10 \%$, and only a very small fraction of the amount administered is able to exert its estrogenic activity. ${ }^{16}$

This estrogenic activity is particularly important when considering the effect at the endometrial level, because progestins reduce nuclear estradiol receptor concentrations, resulting in an antiproliferative effect due to diminished DNA synthesis. In addition, in response to progestins, estradiol is rapidly oxidized to estrone by endometrial cell enzymes, a factor that may exert negative effects on the bleeding profile when estradiol is the estrogenic component of the combined OC. ${ }^{17}$
The transformation of the endometrium in the secretory phase depends on progestational activity and the pharmacokinetics of each progestin. Combined OCs containing estradiol and norethisterone or norethisterone acetate resulted in poor bleeding control. However, combined OCs containing estradiol and dienogest or desogestrel led to better control compared with the combined OCs with estradiol and norethisterone or norethisterone acetate. In addition, the type of regimen used with the combined OCs has been shown to be important, and different trials have reported that monophasic, biphasic, and triphasic combined OCs containing estradiol are consistently associated with abnormal bleeding profiles. ${ }^{13}$

Therefore, new progestins with weak or no androgenic action were developed in an attempt to identify the ideal progestin. ${ }^{18}$ One of these initiatives included the development of dienogest, which was heralded as a new perspective in combined oral contraception. When combined with estradiol, dienogest has been shown to offer optimal endometrial protection and endometrial stability, with good bleeding control when used in a particular four-phasic combination. This new formulation, which contains different doses of estradiol valerate (in a stepdown regimen) and dienogest (in a stepup regimen), was found to result in better bleeding patterns. ${ }^{19}$

\section{Pharmacodynamics}

Dienogest was synthesized by Jena Pharm in 1976 and is a 19-nortestosterone-derived progestogen with a $17 \alpha-$ cyanomethyl group instead of a $17 \alpha$-ethinyl group. ${ }^{20-22}$ Dienogest has a unique pharmacokinetic and pharmacologic profile, combining some of the properties of 19-nortestosterone derivatives with those of progesterone derivatives. ${ }^{20,21}$ In contrast with other 19-nortestosterone derivatives, dienogest has no estrogenic, antiestrogenic, or androgenic activity, but has strong antiandrogenic properties. $^{23}$

Estradiol valerate is an esterified form of natural $17 \beta-\mathrm{E}_{2}$, the most potent endogenous human ovarian estrogen, and is promptly hydrolyzed to estradiol following oral administration. ${ }^{24}$ The mechanism of action of estrogen depends on the estrogen receptor present in the nucleus. The receptor interacts with the specific nucleotide sequences present in target genes. The receptor-estrogen complex binds to DNA and stabilizes a multiprotein complex that includes RNA polymerase and the other proteins necessary for initiating RNA synthesis. ${ }^{24}$ Dienogest, a potent progestogen, has poor binding affinity for estrogen, glucocorticoid, mineralocorticoid, and androgen receptors in vitro, and has been shown to exert an antiandrogenic effect in vitro and in vivo. ${ }^{23,25}$ 


\section{Pharmacokinetics Estradiol valerate}

Following oral administration, estradiol valerate is rapidly hydrolyzed as a result of a metabolic process in the gastrointestinal tract and liver. ${ }^{26}$ Estradiol is further metabolized to estrone and estrone sulfate, which are the major metabolites of estradiol..$^{27,28}$ An open-label, nonrandomized Phase I study of a four-phasic regimen of estradiol valerate-dienogest, in which 15 women underwent treatment over a four-week period and were then followed up for a further three weeks, showed that estradiol concentrations remained stable throughout treatment. The authors concluded that initiating the treatment with a higher dose of estradiol valerate $(3 \mathrm{mg}$ for two days without dienogest) resulted in a steady-state concentration of estradiol and ensured estrogen predominance in the initial days of the cycle. In addition, estradiol concentrations throughout treatment remained within the normal range for the early follicular phase of a spontaneous menstrual cycle (median $31 \mathrm{pg} / \mathrm{mL}$ ), suggesting that all estrogen-dependent functions were maintained. ${ }^{29}$

\section{Dienogest}

Dienogest has its own distinctive properties. It exerts no remarkable estrogenic effects, has a moderate antigonadotropic and potent antiproliferative effect, and shows some antiandrogenic activity. ${ }^{27}$ The pharmacokinetics of oral dienogest are linear (ie, proportional to the dose),,$^{23,25}$ and the oral bioavailability of dienogest is almost $90 \%$. Dienogest is eliminated relatively rapidly, the terminal elimination half-life of the drug being 7.5-8.9 hours after single oral doses of $2-8 \mathrm{mg}$ of dienogest or $2 \mathrm{mg}$ of dienogest plus $30 \mu \mathrm{g}$ ethinylestradiol. ${ }^{25}$ When administered alone, the maximum serum concentrations of dienogest after single and multiple doses were $23 \mathrm{ng} / \mathrm{mL}$ and $32 \mathrm{ng} / \mathrm{mL}$, respectively.
Clearance was around $4 \mathrm{~L} /$ hour after a single dose and remained unchanged with multiple doses of dienogest when administered alone. ${ }^{22}$

\section{Efficacy}

Three studies have investigated the efficacy of the formulation containing different doses of estradiol valerate (stepdown schedule) and dienogest (stepup schedule) in a four-phase combined OC combination (see Figure 1). Inclusion criteria were similar in the three studies, ie, healthy, nonobese, nonsmoking women over 18 years of age. In the first open, noncomparative multicenter European study involving 50 centers in Germany, Austria, and Spain in 2004-2006, a total of 1377 women 18-50 years of age received the study medication for 20 cycles. Thirteen pregnancies occurred over a total exposure time of 23,368 cycles (Pearl Index 0.73, 95\% confidence interval [CI]: 1.24), with six of these pregnancies being attributed to method failure (adjusted Pearl Index 0.34, 95\% CI: 0.73 ). Taking into consideration only the group of women aged $18-35$ years $(n=998), 12$ pregnancies occurred over a total exposure of 16,608 cycles (Pearl Index 0.94, two-sided 95\% CI: 1.65). Five of these pregnancies were attributed to method failure (adjusted Pearl Index 0.40, 95\% CI: 0.92). ${ }^{30}$

A second randomized, comparative, double-blind European trial was conducted to assess bleeding patterns, cycle control, and safety. However, an additional objective was to compare the efficacy of the four-phase estradiol valerate-dienogest combination with that of an ethinylestradiol-levonorgestrel combination. The study included 798 women aged $18-50$ years, with 399 women allocated to each group and then followed up for seven cycles. Study participants were recruited at 34 centers in Germany, the Czech Republic, and France in 2005-2006. One pregnancy occurred in the group of women who completed

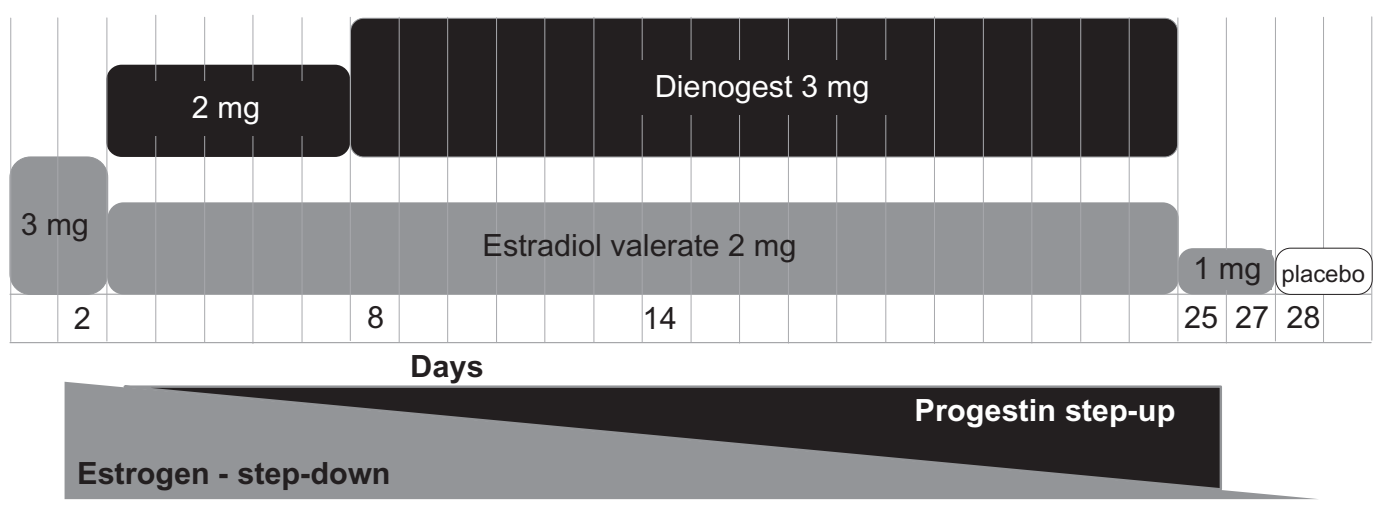

Figure I Dose regimen for the combined oral contraceptive containing estradiol valerate-dienogest. Permission granted. Adapted from summary of product characteristics for Qlaira ${ }^{\circledR} .43$ 
the study using the ethinylestradiol-levonorgestrel combined OC $(n=362)$ compared with none in the group of 365 women who completed the study using the estradiol valerate-dienogest combined OC. ${ }^{19}$

A third open-label, noncomparative study of the fourphase combined OC was conducted in 490 American and Canadian women aged 18-35 years. Follow-up ranged from 13 to 28 cycles, and the number of failures was evaluated according to the validity criterion used by the US Food and Drug Administration, ie, unwanted pregnancies in cycles 1-13 until 14 days after the end of the treatment. Although the study failed to report the total number of cycles evaluated, seven pregnancies occurred, six of which were attributed to method failure. ${ }^{31}$

In the latter study, the authors assessed the overall failure rate of the drug by adding the entire population of women aged 18-35 years in the two European studies and the US study, according to the failures that occurred in cycles 1-13 up to 14 days after discontinuation of the medication. Sixteen pregnancies occurred in 1267 women-years of exposure, resulting in a Pearl Index of 1.27, 95\% CI: 2.06 . For the nine pregnancies attributed to method failure over 1247 women-years of exposure, the adjusted Pearl Index was 0.72 , 95\% CI: $1.37 .{ }^{31}$ These three studies assessing the contraceptive effectiveness of the four-phase estradiol valerate-dienogest combined OC showed the efficacy of the method to be high, comparable with that of the methods containing ethinylestradiol.

\section{Safety and tolerability}

The first European study, which involved 1377 women, reported 59 serious adverse events occurring in 43 women during the study. Two deaths unrelated to the treatment under evaluation were recorded during the study period (accident, intracranial aneurysm). Five serious adverse events were considered by either the investigator or the study sponsor to be at least possibly related to the study medication, including presumed ocular histoplasmosis syndrome, uterine leiomyoma, focal nodular hyperplasia of the liver, myocardial infarction, and deep vein thrombosis. ${ }^{30}$

Additionally, the comparative study reported eight serious adverse events found in seven women during the evaluation period. Five of these events occurred in the estradiol valerate-dienogest group $(n=365)$, and comprised a ruptured ovarian cyst, autonomic nervous system imbalance, vulval abscess, chronic tonsillitis, and renal colic. The three serious adverse events in the ethinylestradiol-dienogest group $(n=362)$ consisted of breast cancer, cholelithiasis, and intervertebral disc prolapse. Of the serious adverse events reported, only the ruptured ovarian cyst and autonomic nervous system imbalance in the estradiol valerate-dienogest group and the case of breast cancer, which occurred in a nonsmoker in the ethinylestradiol-levonorgestrel group who was 30 years old at screening and diagnosed with breast cancer three months after the end of the study, were considered to be possibly related to the treatment. No deaths were reported during the study. ${ }^{19}$ Both studies ${ }^{19,30}$ showed the method to be well tolerated, with almost $80 \%$ of the women stating that they were satisfied or very satisfied with the method, and a discontinuation rate for adverse events of only $10 \%{ }^{19,30,31}$

\section{Bleeding and cycle control}

To improve the tolerability of combined OCs as a contraceptive method, ethinylestradiol was substituted with estradiol valerate. However, the initial regimens (monophasic and biphasic) that were evaluated resulted in unacceptable cycle control and high discontinuation rates. The European trial $^{19}$ compared subjects who received estradiol valeratedienogest (in the dynamic dosing regimen comprising an estrogen stepdown and progestin stepup schedule) or a low-dose combined OC formulation of ethinylestradiol $20 \mu \mathrm{g}+$ levonorgestrel $100 \mu \mathrm{g}$. This study assessed cycle control, and the endpoints evaluated included the incidence and characteristics of scheduled and unscheduled (breakthrough) bleeding in two distinct periods. Bleeding pattern parameters were assessed over 90-day reference periods according to World Health Organization (WHO) recommendations. ${ }^{32}$

Over an evaluation period of seven cycles, users of the combined OC containing estradiol valerate-dienogest reported fewer bleeding/spotting days compared with the women who received a combined OC containing ethinylestradiollevonorgestrel. At the end of the first 90-day period, women reported a mean of $17.3 \pm 10.4$ days of bleeding/spotting (median 16.0) compared with $21.5 \pm 8.6$ (median 21.0) days $(P<0.0001)$ in the estradiol valerate-dienogest group and ethinylestradiol-levonorgestrel groups, respectively. In the second period of the study (days 91-180), women in the estradiol valerate-dienogest group also had significantly fewer bleeding/spotting days, with a mean of $13.4 \pm 9.3$ days (median 12.0 days) compared with $15.9 \pm 7.1$ days (median 15.0 days) in the ethinylestradiol-levonorgestrel group $(P<0.0001)$. The proportion of the women who experienced scheduled withdrawal bleeding per cycle ranged from $77.7 \%$ to $83.2 \%$ and from $89.5 \%$ to $93.8 \%$ in the estradiol 
valerate-dienogest and ethinylestradiol-levonorgestrel groups, respectively.

Most of the women in both groups experienced an absence of scheduled withdrawal bleeding at some point. The group of users of the estradiol valerate-dienogest combined OC experienced an absence of scheduled withdrawal bleeding more often than women using the ethinylestradiol-levonorgestrel combined OC (21.2\% versus $22.2 \%$ of users on one occasion and $12.2 \%$ versus $7.1 \%$ on two occasions). Although $56.9 \%$ of women treated with the estradiol valerate-dienogest combination and $37.8 \%$ of women treated with the ethinylestradiollevonorgestrel combined OC reported an absence of scheduled withdrawal bleeding at least once during the seven cycles of treatment, only a minority $(14.8 \%$ of women in the estradiol valerate-dienogest group and $5.1 \%$ in the ethinylestradiol-levonorgestrel group) reported an absence of scheduled withdrawal bleeding in more than three of the seven treated cycles. Very few women experienced no bleeding at all during the seven cycles $(3.3 \%$ in the estradiol valerate-dienogest group and $0.5 \%$ of the women treated with ethinylestradiol-levonorgestrel).

Scheduled withdrawal bleeding episodes were shorter and lighter in women treated with estradiol valerate-dienogest compared with those treated with ethinylestradiollevonorgestrel. The mean duration of withdrawal bleeding ranged from 4.1 to 4.7 days (median 4.0 days) in women treated with estradiol valerate-dienogest and from 5.0 to 5.2 days (median 5.0 days) in women treated with ethinylestradiol-levonorgestrel $(P<0.05$ per cycle for comparison of the mean values). In addition, the percentage of women reporting unscheduled breakthrough bleeding was similar in both groups, occurring in $14 \%$ and $12 \%$ of women treated with estradiol valerate-dienogest and ethinylestradiollevonorgestrel, respectively (range 9.9\%-17.1\%), with the highest rate occurring in the first cycle of treatment in both groups. Spotting was the predominant form of breakthrough bleeding in both the estradiol valerate-dienogest and ethinylestradiol-levonorgestrel combined OC users; however, breakthrough bleeding was heavy in cycles 1-7 in $2.4 \%$ and $4.0 \%$ of women, respectively. ${ }^{19}$

The analysis involving the three large studies in North America and Europe, which evaluated efficacy over 13 treatment cycles, reported similar results to those of the European study. The mean duration of withdrawal bleeding was 4.0-4.6 days in cycles $1-13$, and the maximum intensity of withdrawal bleeding was mostly described as spotting or light bleeding. The proportion of women who experienced no withdrawal bleeding per cycle ranged from $19.0 \%$ to $24.0 \%$, while breakthrough bleeding tended to decrease between cycles 2 and $13 .{ }^{31}$

\section{Heavy menstrual bleeding}

Combined OCs are commonly used to reduce heavy menstrual bleeding, despite the fact that the relevant data remain inconclusive, and there are no labeling indications for this practice. ${ }^{33}$ Although heavy menstrual bleeding does not lead to anemia or ferritin loss in all women, it does impair quality of life and exerts a detrimental effect on the health, productivity, and well-being of many women. Additionally, it is widely known that women's perception of menstrual blood loss may differ from their actual blood loss; hence not all women with heavy menstrual bleeding seek treatment for the condition. For this reason, the National Institute of Clinical Excellence in the UK has drawn up a subjective definition of heavy menstrual bleeding, which is described as "excessive menstrual blood loss which interferes with the woman's physical, emotional, social and material quality of life, and which can occur alone or in combination with other symptoms". 34

Women over 40 years of age have a high risk of developing menstrual disorders. Conservative treatment was found to be more advantageous than hysterectomy in view of the morbidity and mortality associated with the latter option, which should be avoided whenever possible. ${ }^{35}$ One of the limiting factors to the use of combined OCs among women over 40 years of age is related to the thrombogenic effect of the ethinylestradiol component of different combined OCs. The new combined OC containing estradiol valerate, a natural estrogen, represents a potentially safe method for women in the menopausal transition. There is now evidence that the dynamic-dosing of the estradiol valerate-dienogest combined OC represents an effective treatment for heavy menstrual bleeding.

Two multicenter, double-blind, randomized, placebocontrolled studies, the designs of which were identical, were conducted in parallel in the US and Canada ${ }^{36}$ and Europe and Australia ${ }^{33}$ to assess the effects of an estradiol valeratedienogest combined OC in women with heavy menstrual bleeding. Using the alkaline hematin method, women over 18 years of age who were found to have either heavy menstrual bleeding (defined as $\geq$ two bleeding episodes with a blood loss volume of $\geq 80 \mathrm{~mL}$ each) and/or prolonged bleeding ( $\geq$ two episodes each lasting $\geq$ eight days) or frequent bleeding ( $>$ five bleeding episodes with a minimum of 20 bleeding days overall) confirmed during a 90-day runin 
period were enrolled in the study. The primary outcome was complete response (defined as restoration of completely normal menstruation) during a 90-day efficacy period versus the 90-day runin period. Other outcomes were menstrual blood loss and iron metabolism. Women were excluded from the study if they had any uterine or systemic disease that could affect menstrual blood loss (eg, fibroids, polyps, endometrial hyperplasia) or systemic diseases, such as von Willebrand disease.

The study conducted in Europe and Australia ${ }^{33}$ assessed the efficacy of the estradiol valerate-dienogest combined OC for the treatment of heavy and/or prolonged menstrual bleeding in women with no evident pathology. Data collected over 90 days of treatment from women receiving estradiol valerate-dienogest $(n=149)$ or placebo $(n=82)$ were compared with data from the 90 -day runin period. The difference in menstrual blood loss over 90 days with estradiol valerate-dienogest was $-373 \mathrm{~mL}$ (95\% CI: $-490,-255$; $P<0.0001)$. Hemoglobin and ferritin levels improved only in the estradiol valerate-dienogest group (median $+0.6 \mathrm{~g} / \mathrm{dL}$, 95\% CI: $0.3-1.0 ; P<0.0001$ and $+8.2 \mathrm{ng} / \mathrm{mL}, 95 \%$ CI: 3.5-12.9; $P=0.002$ ), respectively. ${ }^{33}$

The trial conducted in North America ${ }^{36}$ included 190 women who were randomized to use estradiol valeratedienogest $(n=120)$ or placebo $(n=70)$. Of these, 136 women completed the study ( 85 in the estradiol valerate-dienogest group and 51 in the placebo group). The authors reported similar results to those of Fraser et al, ${ }^{33}$ with a significantly higher complete response rate in users of the combined OC containing estradiol valerate-dienogest $(43.8 \%, 95 \% \mathrm{CI}$ : $32.7 \%-55.3 \%)$ compared with that found in the placebo group (4.17\%, 95\% CI: $0.5 \%-14.3 \%)$. Furthermore, the mean reduction in menstrual blood loss was significantly greater in users of the estradiol valerate-dienogest combination compared with the placebo group $(-353$ versus $-130 \mathrm{~mL}$; $P<0.0001)$. Menstrual blood loss decreased in women in the estradiol valerate-dienogest group from cycle 2 onwards and hemoglobin, hematocrit, and ferritin levels improved significantly; however, there was no change in these parameters in the group receiving placebo.

The methodology was rigorous in both studies, and both used the alkaline hematin method, which is considered the gold standard for measurement of menstrual blood loss and for defining heavy menstrual bleeding. ${ }^{37}$ These results were encouraging, and provide a new insight into the use of a combined OC for the effective treatment of idiopathic heavy menstrual bleeding.

\section{Place in therapy}

The combined OC containing estradiol valerate-dienogest, administered to healthy women in a four-phase dosing regimen (with an estrogen stepdown and a progestin stepup schedule over 26 days), was shown to be a highly effective, safe, and well tolerated hormonal contraceptive. Its positive profile, which includes characteristics of lack of any significant effect on the cardiovascular system and good cycle control, with a tendency towards a decrease in bleeding, makes it an excellent clinical option for women over 40 years of age who need contraception and/or for control of heavy menstrual bleeding or dysfunctional bleeding in the menopausal transition. In addition, its use could possibly be extended to postmenopausal women as hormone therapy.

Although extended use of the progestin phase beyond 21 days did not appear to result in substantial benefits, extended use of the estrogen component could offer promising advantages. The most important feature of this new combined OC containing estradiol valerate-dienogest, administered in a four-phase dosing regimen, may lie in providing a better estrogen to progestin ratio at every phase of the cycle. The local effects of estradiol would be balanced by the antiproliferative effects of the progestin. The estrogen dominance in the first days of use, and consequently at the beginning of follicular development, may induce estradiol and progesterone receptors, which could support proliferation of the endometrium, in turn improving endometrial responsiveness to the progestin, endometrial stroma stability, and cycle control. ${ }^{13}$

The results from the two clinical trials conducted to test this novel combination in women suffering from heavy menstrual bleeding showed that estradiol valerate-dienogest is a well tolerated combined OC with excellent cycle control. Although withdrawal bleeding tends to be lighter and shorter compared with that found with other combined OCs, more women using the estradiol valerate-dienogest combined OC did not experience withdrawal bleeding than those using a traditional 21/7-day combined OC, ${ }^{19}$ and this issue needs to be studied carefully to evaluate the acceptability of this feature for women.

Although it is obvious that estradiol has better metabolic effects than ethinylestradiol, the available data were obtained from surrogate studies ${ }^{38}$ and are insufficient to enable any conclusion to be drawn with respect to the increased safety of the combined OC containing estradiol valerate-dienogest compared with existing combined OCs. Consequently, it should be taken into account that the eligibility criteria 
established by the $\mathrm{WHO}^{39}$ for the use of combined OCs are also valid for this new pill, and caution must be exercised until further evidence is found to change this situation.

This new combined OC has been approved in some European countries for contraception only. However, the favorable profile observed in women with heavy menstrual bleeding and the acceptable menstrual blood loss achieved with the method suggest that it may represent an excellent new option for the treatment of this disorder, bearing in mind that many women with heavy menstrual bleeding also need a reliable and effective contraceptive method. Hopefully, regulatory authorities will approve the use of this combined OC for heavy menstrual bleeding, thereby providing physicians and women with a new option that may expand the choices already available for the treatment of this problem, particularly considering that many women prefer not to undergo surgery for heavy menstrual bleeding. ${ }^{40}$

There are plenty of brands and compositions of combined OCs on the market, and simply adding one more to the already available arsenal may fail to impress many health providers and their clients. The main question to be considered is, if the current combined OC used by the patient works well, why should she change to a new one, the cost of which may be higher than that of her current pill? However, it should be taken into consideration that, as women grow older, they are also more likely to experience bleeding problems at a time when, in many cases, pregnancy is unwelcome. Women in the menopausal transition are more likely to experience bleeding problems, but still require a highly effective contraceptive method. This new combined OC may offer both advantages.

In view of current concerns with respect to ethinylestradiol, the most important innovation with this new combined OC is the replacement of ethinylestradiol by estradiol, and in this specific case, by estradiol valerate. In oral compounds, estradiol stimulates the liver during the initial first pass, and the overall impact is lower than that found with a biologically equivalent dose of ethinylestradiol. ${ }^{41}$ Nevertheless, large epidemiologic studies need to be conducted in populations of patients of different age groups, with different body mass indexes, of different ethnic groups, and with different risk factors to evaluate whether estradiol-containing combined OCs will reduce the risk of thrombosis that exists with current combined OCs. A Phase IV safety study of the estradiol valerate-dienogest combined OC (International Active Surveillance Study of Women Taking Oral Contraceptives) $)^{42}$ has already begun and will provide evidence on the safety of this pill.

\section{Conclusion}

This is the first estradiol-containing combined OC on the market, and it appears to provide good contraceptive efficacy and excellent cycle control, with a confirmed reduction in menstrual blood loss in women with heavy menstrual bleeding. Although contraceptive methods were initially designed to prevent pregnancy, there is an increasingly accepted concept that they should offer additional noncontraceptive benefits. This new combined OC offers the advantage of reducing menstrual blood loss, and represents a valid treatment for heavy menstrual bleeding. The advantage of having estradiol in its composition represents a new option for many women, in particular for those with heavy menstrual bleeding.

\section{Disclosure}

The authors report no conflicts of interest in this work.

\section{References}

1. Gestodene Study Group 322. The safety and contraceptive efficacy of a 24-day low dose oral contraceptive regimen containing gestodene $60 \mu \mathrm{g}$ and ethinylestradiol $15 \mu \mathrm{g}$. Eur J Contracept Reprod Health Care. 1999;4 Suppl 2:9-15.

2. Bachmann G, Sulak PJ, Sampson-Landers C, et al. Efficacy and safety of a low-dose 24-day combined oral contraceptive containing $20 \mu \mathrm{g}$ ethinylestradiol and $3 \mathrm{mg}$ drospirenone. Contraception. 2004;70(3): 191-198.

3. Gallo MF, Nanda K, Grimes DA, Schulz KF. 20 mcg versus $>20$ mcg estrogen combined oral contraceptives for contraception. Cochrane Database Syst Rev. 2005;2:CD003989.

4. Cibula D, Karck U, Weidenhammer HG, Kunz J, Alincic S, Marr J. Efficacy and safety of a low-dose 21-day combined oral contraceptive containing ethinylestradiol 20 microg and drospirenone $3 \mathrm{mg}$. Clin Drug Investig. 2006;26(3):143-150.

5. Bonnar J. Coagulation effects of oral contraception. Am J Obstet Gynecol. 1987;157(4 Pt 2):1042-1048.

6. Bonnar J, Daly L, Carroll E. Blood coagulation with a combination pill containing gestodene and ethinyl estradiol. Int J Fertil. 1987;32 Suppl: $21-28$.

7. Sabra A, Bonnar J. Hemostatic system changes induced by 50 micrograms and 30 micrograms estrogen/progestogen oral contraceptives. Modification of estrogen effects by levonorgestrel. J Reprod Med. 1983;28 Suppl 1:85-91.

8. World Health Organization Task Force on Oral Contraception. A randomized double-blind study of two combined oral contraceptives containing the same progestogen, but different estrogens. Contraception. 1980;21(5):445-459.

9. Serup J, Bostofte E, Larsen S, Westergaard J, Lebech PE. Natural oestrogens for oral contraception. Lancet. 1979;2(8140):471-472.

10. Kivinen S, Saure A. Efficacy and tolerability of a combined oral contraceptive containing 17 beta-estradiol and desogestrel. Eur J Contracept Reprod Health Care. 1996;1:183.

11. Hirvonen E, Stenman UH, Malkonen M, et al. New natural oestradiol/ cyproterone acetate oral contraceptive for pre-menopausal women. Maturitas. 1988;10(3):20120-13.

12. Wenzl R, Coeling Bennink H, van Beek A, et al. Ovulation inhibition with a combined oral contraceptive containing $1 \mathrm{mg}$ micronized 17 betaestradiol. Fertil Steril. 1993;60(4):616-619.

13. Fruzzetti F, Bitzer J. Review of clinical experience with estradiol in combined oral contraceptives. Contraception. 2010;81(1):8-15. 
14. Kuhnz W, Blode H, Zimmerman H. Pharmacokinetics of exogenous natural and synthetic estrogens and antiestrogens. In: Oettel M, Schillinger E, editors. Handbook of Experimental Pharmacology. Berlin: Springer; 1999.

15. Longcope C, Williams KI. The metabolism of estrogens in normal women after pulse injections of $3 \mathrm{H}$-estradiol and $3 \mathrm{H}$-estrone. J Clin Endocrinol Metab. 1974;38(4):602-607.

16. Dusterberg B, Nishino Y. Pharmacokinetic and pharmacological features of oestradiol valerate. Maturitas. 1982;4(4):315-324.

17. Hoffmann H, Moore C, Kovacs L, et al. Alternatives of the replacement of ethinylestradiol by natural 17 beta-estradiol in dienogest-containing oral contraceptives. Drugs Today (Barc). 1999;35:105-113.

18. Wiegratz L, Lee, JH, Kutschera E, et al. Effect of dienogestcontaining oral contraceptives on lipid metabolism. Contraception. 2002;65(3):223-229.

19. Ahrendt HJ, Makalová D, Parke S, Mellinger U, Mansour D. Bleeding pattern and cycle control with an estradiol-based oral contraceptive: A seven-cycle, randomized comparative trial of estradiol valerate/ dienogest and ethinyl estradiol/levonorgestrel. Contraception. 2009; 80(5):436-444.

20. Oettel M, Bervoas-Martin S, Elger W, et al. A 19-norprogestin without $17 \alpha$-ethinyl group II: Dienogest from a pharmacokinetic point of view. Drugs Today (Barc). 1995;31(7):499-516.

21. Oettel M, Holz C. Hybrid progestins: The example of dienogest. In: Sitruk-Ware R, Mishell DR, editors. Progestins and Antiprogestins in Clinical Practice. New York, NY: Marcel Dekker; 2000.

22. Zimmermann H, Duvauchelle T, Gualano V, et al. Pharmacokinetics of dienogest as single drug or in combination with estradiol valerate or ethinylestradiol. Drugs Today (Barc). 35 Suppl C:27-39.

23. Lievertz RW. Pharmacology and pharmacokinetics of estrogens. Am J Obstet Gynecol. 1987;156(5):1289-1293.

24. Graser T, Hoffman H, Zimmerman H, et al. Lafamme ${ }^{\circledR}$ : A new oral preparation for continuous combined hormone replacement therapy in postmenopausal women. Drugs Today (Barc). 2001;37 Suppl G: $12-27$.

25. Oettel M, Carol W, Elger W, et al. A 19-norprogestin without a 17 alpha-ethinyl group. II: Dienogest from a pharmacodynamic point of view. Drugs Today (Barc). 1995;31(7):517-536.

26. Endrikat J, Parke S, Trummer D, et al. Ovulation inhibition with four variations of a four-phasic estradiol valerate/dienogest combined oral contraceptive: Results of two prospective, randomized, open-label studies. Contraception. 2008;78(3):218-225.

27. Oettel M, Breitbarth H, Elger W, et al. The pharmacological profile of dienogest. Eur J Contracept Reprod Health Care. 1999;4 Suppl 1: $2-13$.

28. Oettel M, Graeser T, Hoffman H, et al. The preclinical and clinical profile of dienogest. A short overview. Drugs Today (Barc). 1999;35 Suppl C: 3-12.

29. Zeun S, Lu M, Uddin A, Zeiler B, Morrison D, Blode H. Pharmacokinetics of an oral contraceptive containing oestradiol valerate and dienogest. Eur J Contracept Reprod Health Care. 2009;14(3):221-232.
30. Palacios S, Wildt L, Parke S, Machlitt A, Römer T, Bitzer J. Efficacy and safety of a novel oral contraceptive based on oestradiol (oestradiol valerate/dienogest): A Phase III trial. Eur J Obstet Gynecol Reprod Biol. 2010;149(1):57-62.

31. Nelson A, Sampson-Landers C, Parke S, Jensen J. Efficacy of estradiol valerate/dienogest OC: Results of 3 large studies in North America and Europe. Abstract and poster presented at the 57th Annual Clinical Meeting of the American College of Obstetricians and Gynecologists, 2009 May 2-6, Chicago, IL.

32. Belsey EM. Vaginal bleeding patterns among women using one natural and eight hormonal methods of contraception. Contraception. 1988;38(2):181-206.

33. Fraser IS, Zeun S, Machlitt A, Mellinger U. A novel oral contraceptive comprising estradiol valerate/dienogest for the treatment of heavy and/ or prolonged menstrual bleeding without organic cause: A double-blind, randomised, placebo-controlled trial. Int J Gynecol Obstet. 2009; 107 Suppl 2:S183.

34. National Collaborating Centre for Women's and Children's Health. Heavy menstrual bleeding. Commissioned by the National Institute for Health and Clinical Excellence. Available from: http://wwwniceorguk/ nicemedia/pdf/CG44FullGuidelinepdf. Accessed 2010 Mar 5.

35. Lethaby A, Shepperd S, Cooke I, et al. Endometrial resection and ablation versus hysterectomy for heavy menstrual bleeding. Cochrane Database Syst Rev. 2000;2:CD000329.

36. Jensen J, Machlitt A, Mellinger U, et al. A multicenter, double-blind, randomized, placebo-controlled study of oral estradiol valerate/denogest for the treatment of heavy and/or prolonged menstrual bleeding. Fertil Steril. 2009;92(3):S32.

37. van Eijkeren MA, Scholten PC, Christiaens GC, Alsbach GP, Haspels AA. The alkaline hematin method for measuring menstrual blood loss - a modification and its clinical use in menorrhagia. Eur $J$ Obstet Gynecol Reprod Biol. 1986;22(5-6):345-351.

38. Grimes DA, Schulz KF, Raymond EG. Surrogate end points in women's health research: Science, protoscience, and pseudoscience. Fertil Steril. 2010;93(6):1731-1734.

39. World Health Organization. Medical Eligibility Criteria for Contraceptive Use. 4th ed. Geneva: World Health Organization; 2009.

40. Jensen JT. Evaluation of new estradiol oral contraceptive: Estradiol valerate and dienogest. Expert Opin Pharmacother. 2010;11(7): 1147-1157.

41. Eilertsen AL, Hoibraaten E, Os I, et al. The effects of oral and transdermal hormone replacement therapy on C-reactive protein levels and other inflammatory markers in women with high risk of thrombosis. Maturitas. 2005;52(2):111-118.

42. ClinicalTrials.gov identifier: NCT01009684.

43. Qlaira ${ }^{\circledR}$. Summary of product characteristics. 2009 Dec 22. Available from: http://emc.medicines.org.uk/medicine/21700/SPC/Qlaira/. Accessed 2010 Sep 29
Open Access Journal of Contraception

\section{Publish your work in this journal}

Open Access Journal of Contraception is an international, peerreviewed, open access, online journal, publishing original research, reports, reviews and commentaries on all areas of contraception. In addition to clinical research, demographics and health-related aspects, the journal welcomes new findings in animal and preclinical studies

\section{Dovepress}

relating to understanding the biological mechanisms and practical development of new contraceptive agents. The manuscript management system is completely online and includes a very quick and fair peer-review system. Visit http://www.dovepress.com/testimonials.php to read real quotes from published authors. 